\title{
MARCHA DE ABSORÇÃO DOS MICRONUTRIENTES PARA MUDAS DE GOIABEIRAS CULTIVARES PALUMA E SÉCULO XXI $\left({ }^{1}\right)$
}

\author{
CLAUDENIR FACINCANI FRANCO $\left({ }^{2}\right)$; RENATO DE MELLO PRADO $\left(3^{*}\right)$; \\ LUIZ FERNANDO BRAGHIROLLI $\left({ }^{2}\right)$; DANILO EDUARDO ROZANE $\left({ }^{2}\right)$
}

\begin{abstract}
RESUMO
O conhecimento da marcha de absorção dos micronutrientes em mudas de goiabeira garante melhor eficiência da adubação. O objetivo do trabalho foi determinar a marcha de absorção de micronutrientes em mudas de duas cultivares de goiabeira. O delineamento experimental utilizado foi em parcelas subdivididas com três repetições. Assim, foram utilizadas como tratamentos principais duas cultivares de goiabeira (Paluma e Século XXI) obtidas por estacas herbáceas e como subparcelas, sete coletas de plantas, ao longo do período experimental (120 dias). Nos diferentes órgãos das mudas (folhas, caule e raízes), avaliou-se o acúmulo de micronutrientes, a cada quinze dias. A muda de goiabeira da cultivar Século XXI é mais exigente em micronutrientes do que as mudas da cultivar Paluma. O acúmulo médio de micronutrientes pelas mudas de goiabeira obedeceu à seguinte seqüência: $\mathrm{Fe}>\mathrm{Mn}>\mathrm{Zn}>\mathrm{B}>\mathrm{Cu}$, sendo maior nas folhas para $\mathrm{B}, \mathrm{Cu}, \mathrm{Mn}$ e $\mathrm{Zn}$, e nas raízes para Fe. O acúmulo de micronutrientes para as cultivares Paluma e Século XXI em $\mu$ g por planta foi de: B (632 e 783), Cu (134 e 158), Fe (8103 e 5534$)$, Mn (3052 e 3709) e Zn (760 e 997) respectivamente.
\end{abstract}

Palavras-chave: Psidium guajava, nutrição mineral, acúmulo de micronutrientes, exigência nutricional, solução nutritiva.

\section{ABSTRACT \\ MICRONUTRIENT UPTAKE IN ‘PALUMA`AND ‘SÉCULO XXI’ GUAVA CUTTINGS}

Understanding micronutrient uptake in guava cuttings guarantees efficient fertilization. This research was undertaken to measure micronutrient uptake in cuttings from two guava cultivars. The experimental design was arranged in split-plots with three replications. Plots were represented by herbaceous cuttings from the guava cultivars 'Paluma' and 'Século XXI', while split-plots were the seven sampling dates during a 120-day period in nutritive solution. Accumulation of micronutrients was determined in the different plant organs every two weeks. The guava cultivar 'Século XXI' has higher micronutrient requirements than 'Paluma'. The mean micronutrient accumulation followed the order: $\mathrm{Fe}>\mathrm{Mn}>\mathrm{Zn}>\mathrm{B}>\mathrm{Cu}$. The accumulation of $\mathrm{B}, \mathrm{Cu}, \mathrm{Mn}$ and $\mathrm{Zn}$ was higher in leaves, while Fe was more accumulated in roots. Micronutrient accumulation in the cultivars 'Paluma' and 'Século XXI', in $\mu \mathrm{g}$ per plant, was (respectively): B: 632 and 783; Cu: 134 and 158; Fe: 8103 and 5534; Mn: 3052 and 3709; and Zn 760 and 997.

Key words: Psidium guajava, mineral nutrition, micronutrient accumulation, nutritional requirement, nutrient solution.

$\left({ }^{1}\right)$ Recebido para publicação em 24 de janeiro e aceito em 19 de abril de 2007.

$\left({ }^{2}\right)$ Pós-Graduando em Agronomia, Departamento de Solos e Adubos, FCAV/Unesp, Campus Jaboticabal. Via de Acesso Paulo Donato Castellane, s/n. ${ }^{\circ}, 14884-900$ Jaboticabal (SP). E-mail: franco@fcav.unesp.br. Bolsista CNPq.

$\left({ }^{3}\right)$ Departamento de Solos e Adubos, FCAV/Unesp, Campus Jaboticabal. Via de Acesso Paulo Donato Castellane, s/n. 14884-900 Jaboticabal-SP. E-mail: rmprado@fcav.unesp.br $\left(^{*}\right)$ Autor correspondente. Bolsista Produtividade em Pesquisa do CNPq. 


\section{INTRODUÇÃO}

O Brasil é o maior produtor de goiaba, mas sua participação no mercado internacional é muito pequena. Em 2002, o país produziu 300 mil toneladas e exportou somente $0,06 \%$ desse volume, sendo essa relação considerada baixa quando comparada à de países tradicionalmente produtores de frutas (ROZANE e Couto, 2003).

Em 2003, a produção nacional de goiabas foi de 329 mil toneladas, ocupando uma área de $18 \mathrm{mil}$ hectares, sendo São Paulo e Pernambuco responsáveis por $67 \%$ da produção nacional (Agrianul, 2006). Em 2005, somente no Estado de São Paulo a produção de goiaba foi de 124 toneladas das quais $46 \%$ foram obtidas da cultivar Paluma (ANUÁRIo IEA, 2006). Para garantir a expansão da goiabeira, a fase de implantação do pomar é fundamental. Nesta fase, a qualidade das mudas é muito importante para garantir a homogeneidade, a rápida formação e o início precoce da produção.

Assim, a produção de mudas de fruteiras está tendo alterações significativas no sistema de produção, existindo uma preocupação para uso de tecnologias de produção com padrão de qualidade, sanidade, menor custo e de fácil obtenção. Atualmente, para produção de mudas de goiabeira tem aumentado o interesse em utilização de substratos inertes esterilizados; entretanto, há baixa concentração de nutrientes e a adubação é importante para a adequada nutrição das mudas. Deste modo, a utilização de substrato inerte para a produção de mudas de goiabeira tem propiciado o emprego da fertirrigação (solução nutritiva), sendo, no entanto realizada de forma empírica pelos viveiristas. Para garantir a maior eficiência da adubação para produção de mudas de goiabeira, torna-se importante conhecer a dinâmica de acúmulo de nutrientes na massa seca e ao longo do tempo de cultivo.

Salienta-se, ainda, que não há informações científicas disponíveis na literatura nacional sobre demanda por nutrientes para mudas de goiabeira, tanto para cultivar Paluma - a mais cultivada do Brasil - como às novas cultivares, como a Século XXI, ambas desenvolvidas na FCAV/Unesp, Câmpus Jaboticabal, sobretudo para micronutrientes que possibilitem sustentar programas de adubação.

É conhecida a importância dos micronutrientes na nutrição de plantas, especialmente como ativador ou constituinte de enzimas e também têm papel estrutural, constituindo compostos orgânicos (Malavolta et al., 1997). Além da importância dos micronutrientes na produção de biomassa, é sabido que a quantidade relativa desses nutrientes aplicada por muda é muito pequena, portanto, tem-se alta relação benefício/custo, conseqüentemente maior sustentabilidade nos sistemas de produção de mudas de frutíferas.

Assim, o objetivo do trabalho foi determinar a marcha de absorção de micronutrientes em mudas de duas cultivares de goiabeira.

\section{MATERIAL E MÉTODOS}

O experimento foi realizado em condições de casa de vegetação, sob hidroponia, na FCAV/Unesp, Campus de Jaboticabal, coordenadas $21^{\circ} 15^{\prime} 22^{\prime \prime}$ Sul, $48^{\circ} 18^{\prime} 58^{\prime \prime}$ Oeste e altitude de 575 metros.

O delineamento experimental utilizado foi em parcelas subdivididas, com três repetições. Assim, foram utilizadas como tratamentos principais duas cultivares de goiabeira (Paluma e Século XXI) e, como subparcelas, sete coletas de plantas ao longo do período experimental.

Foram utilizadas plantas obtidas a partir de propagação vegetativa de estacas herbáceas de matrizes selecionadas de goiabeiras (Psidium guajava L.). As estacas, compostas de um segmento de haste (cerca $12 \mathrm{~cm}$ de comprimento) com um par de folhas, correspondendo a um internódio, foram inicialmente colocadas em caixas de madeira contendo vermiculita, recebendo nebulização intermitente de água, a cada 15 segundos, por um período de 90 dias, até enraizarem.

Após o enraizamento inicial, as estacas tiveram a metade de cada uma das folhas cortadas, bem como redução do sistema radicular. Em seguida, as mudas foram transplantadas e, durante os primeiros 15 dias, foram mantidas em solução nutritiva completa, diluída a $1 / 4$, com cinco plantas por vaso para posterior seleção (desbaste), deixando quatro plantas por unidade experimental, composta por um vaso de polipropileno $(8 \mathrm{~L})$, com dimensões de $48 \mathrm{~cm}$ de comprimento $\times 16 \mathrm{~cm}$ de largura $\times 17 \mathrm{~cm}$ de altura. Após este período, as plantas foram submetidas à solução nutritiva completa, até 120 dias após o transplantio.

A solução utilizada foi a de Castellane e ARAújo (1995), indicada como adequada para cultivo de mudas de goiabeira segundo Franco e Prado (2006), com as seguintes concentrações de nutrientes em mg L-1 $, \mathrm{N}=222,5 ; \mathrm{P}=61,9 ; \mathrm{K}=426,2 ; \mathrm{Ca}=139,9$; $\mathrm{Mg}=24,3 ; \mathrm{S}=32,4$, e em $\mu \mathrm{g} \mathrm{L}^{-1}, \mathrm{~B}=498 ; \mathrm{Cu}=48 ; \mathrm{Fe}$ $=5000 ; \mathrm{Mn}=419 ; \mathrm{Mo}=52 ; \mathrm{Zn}=261$ de solução nutritiva respectivamente. 
As soluções nutritivas foram mantidas em aeração constante, com o uso de borbulhador acoplado a um compressor de ar. $\mathrm{O}$ valor do $\mathrm{pH}$ da solução nutritiva foi monitorado diariamente, com o uso de um peagômetro portátil (PG 1400), e ajustado a 5,5 $\pm 0,5$, utilizando-se de solução $\mathrm{NaOH}$ ou $\mathrm{HCl} 0,1 \mathrm{~mol} \mathrm{~L}^{-1}$. Na mesma ocasião, foi monitorada a condutividade elétrica da solução nutritiva, com um condutivímetro portátil (CG220), mantendo-a com valor inferior a 2,4 ds $\mathrm{m}^{-1}$, conforme indicação de TÁvorA et al. (2001) para o cultivo de mudas de goiabeira.

Utilizou-se água deionizada para compor a solução nutritiva e para a reposição da água evapotranspirada. A solução nutritiva foi renovada quinzenalmente.

A primeira coleta das plantas foi realizada 30 dias após transplantio e, conseqüentemente, as plantas desta coleta estavam em contato com a solução nutritiva completa durante os primeiros 15 dias. A partir dessa coleta, foram realizadas, quinzenalmente, as demais coletas de plantas até completar os 120 dias de transplantio.

A cada coleta, as plantas foram divididas em raízes, caule e folhas. Depois, todo o material vegetal foi lavado em água deionizada e seco em estufa com circulação forçada de ar, à temperatura de 65 a $70^{\circ} \mathrm{C}$, até atingir massa constante. Foi quantificada a massa da matéria seca das diferentes partes da planta e, em seguida, moída e armazenada. Na seqüência, determinou-se o teor de micronutrientes no tecido vegetal, seguindo método descrito por BATAGLIA et al. (1983). A partir do teor de nutrientes e da massa seca, calculou-se o acúmulo de micronutrientes nos diferentes órgãos das mudas de goiaba.

Com base nos resultados para as variáveis estudadas, foram realizadas análises de variância (teste F) para comparação entre as cultivares de goiabeira, e à análise de regressão para tempo de cultivo.

\section{RESULTADOS E DISCUSSÃO}

Observou-se que o houve incremento no acúmulo de matéria seca tanto da cultivar Paluma (P), como para a Século XXI (S), em função do tempo de cultivo, nos diferentes órgãos e que refletiu na planta inteira (Figura 1). Na cultivar Século XXI, observou-se maior acúmulo de massa seca nos diferentes órgãos, exceto raiz, comparada com a cultivar Paluma (Tabela 1).

Com relação à nutrição das plantas, observa-se que apenas os teores foliares de $\mathrm{Cu}, \mathrm{Mn}$ e $\mathrm{Zn}$ foram significativos para o fator cultivar. Na cultivar Paluma o teor foliar foi superior à 'Século XXI' para Mn e Zn, enquanto para o Cu este comportamento foi oposto (Tabela 2). Em 'Paluma' houve aumento linear para teor de Mn e quadrático para os teores de $\mathrm{B}, \mathrm{Cu}$, Fe e Zn, enquanto na cultivar Século XXI, notou-se variação quadrática para todos os micronutrientes, ao longo do período experimental (Tabela 3). Observa-se que o aumento no teor dos micronutrientes das folhas das mudas, em função do tempo, foi semelhante ao acúmulo de matéria seca de folhas. Portanto, não houve efeito diluição, que pode ocorrer durante o crescimento inicial das plantas.

É pertinente salientar que os teores de micronutrientes ao longo do cultivo, quando substituído na equação da tabela 3 os valores de $x=30$ e 120 dias tem-se como o B (25 a $\left.60 \mathrm{mg} \mathrm{kg}^{-1}\right)$; Mn (114 a $147 \mathrm{mg} \mathrm{kg}^{-1}$ ) e o $\mathrm{Fe}\left(68\right.$ a $\left.154 \mathrm{mg} \mathrm{kg}^{-1}\right)$, sendo obtidos teores acima da faixa considerada adequada por NATALE et al. (1996) (B = 20 a $25 ; \mathrm{Mn}=114$ a 147; $\left.\mathrm{Fe}=60-90 \mathrm{mg} \mathrm{kg}^{-1}\right)$, exceto o Fe aos 30 dias após o transplantio. O teor de $\mathrm{Cu}\left(4 \mathrm{a} 9 \mathrm{mg} \mathrm{kg}^{-1}\right)$, está baixo e o Zn (29 a $37 \mathrm{mg} \mathrm{kg}^{-1}$ ) (Tabela 3), praticamente na faixa adequada segundo os mesmo autores $(\mathrm{Cu}=20$ a 40 $\mathrm{e} Z \mathrm{n}=25$ a $35 \mathrm{mg} \mathrm{kg}^{-1}$ ). Entretanto, observa-se que os teores de micronutrientes dos tecidos vegetais deste trabalho, comparados com a literatura são distintos, pois NATALE et al. (1996) utilizaram o $3 .^{\circ}$ par de folhas de plantas adultas e neste trabalho são as folhas da parte aérea de plantas jovens. Assim, a comparação dos resultados do trabalho com a literatura fica prejudicado, devido à ausência de informações na literatura sobre o teor de nutrientes adequados em mudas de goiabeira.

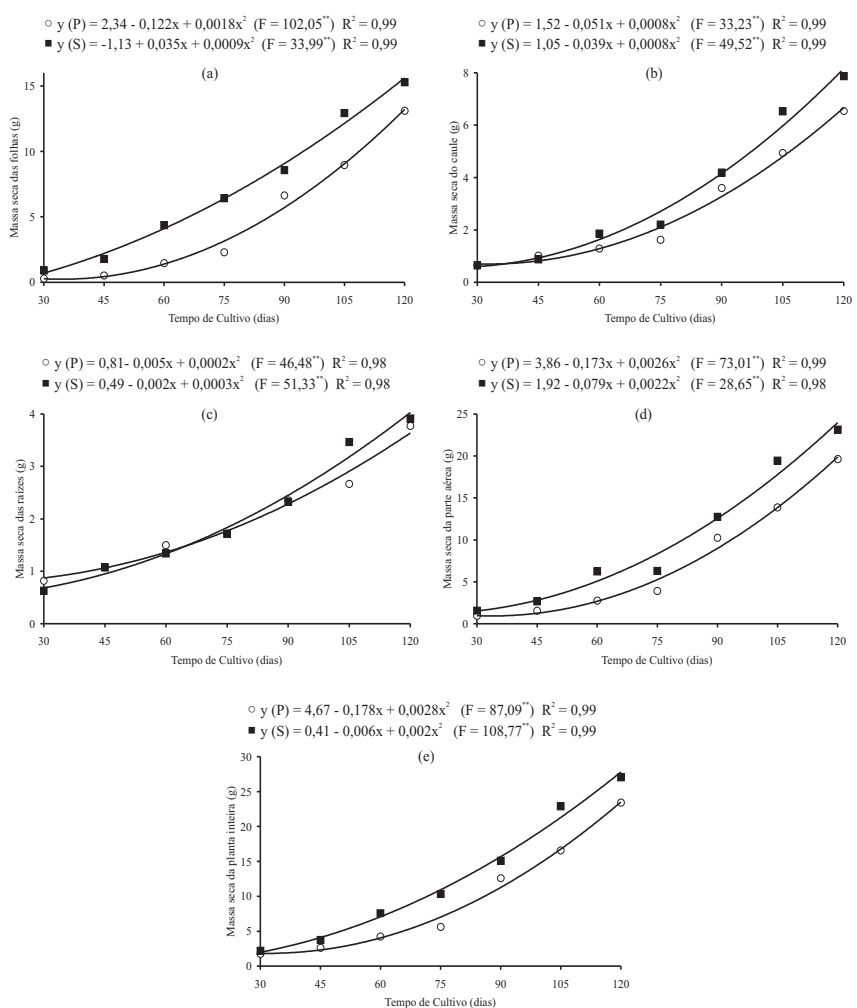

Figura 1. Efeito do tempo de cultivo no acúmulo de massa seca das folhas (a), do caule (b), das raízes, (c) da parte aérea (d) e da planta inteira (e) das mudas de goiabeira cultivares Paluma (P) e Século XXI (S). 
Tabela 1. Valor de $\mathrm{F}$ e os dados médios do fator cultivar e do tempo de cultivo das mudas de goiabeira sobre as variáveis de crescimento das plantas cultivadas em solução nutritiva

\begin{tabular}{|c|c|c|c|c|c|}
\hline Fatores & $\begin{array}{l}\text { Massa seca } \\
\text { das folhas }\end{array}$ & $\begin{array}{c}\text { Massa seca } \\
\text { do caule }\end{array}$ & $\begin{array}{c}\text { Massa seca } \\
\text { das raízes }\end{array}$ & $\begin{array}{c}\text { Massa seca } \\
\text { da parte aérea }\end{array}$ & $\begin{array}{c}\text { Massa seca } \\
\text { total }\end{array}$ \\
\hline & & & - Teste F & 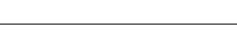 & 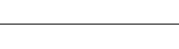 \\
\hline Cultivar (C) & $181,55^{* *}$ & $45,31^{* *}$ & $5,63^{\mathrm{ns}}$ & $45,69^{* *}$ & $254,05^{* *}$ \\
\hline Tempo de cultivo(T) & $469,01^{* *}$ & $174,15^{* *}$ & $448,10^{* *}$ & $223,01^{* *}$ & $540,79^{* *}$ \\
\hline Interação (CxT) & $7,75^{*}$ & $2,68^{*}$ & $10,02^{* *}$ & $2,53^{*}$ & $7,26^{* *}$ \\
\hline Paluma & $4,75 \mathrm{~b}$ & $2,81 \mathrm{~b}$ & 1,99 & $7,55 \mathrm{~b}$ & $9,54 \mathrm{~b}$ \\
\hline Século XXI & $7,20 \mathrm{a}$ & $3,45 \mathrm{a}$ & 2,07 & $10,30 \mathrm{a}$ & $12,69 \mathrm{a}$ \\
\hline C.V. $(\%)^{1}$ & 9,8 & 9,9 & 5,4 & 14,7 & 5,8 \\
\hline C.V. $(\%)^{2}$ & 9,8 & 15,1 & 6,4 & 14,3 & 8,3 \\
\hline
\end{tabular}

ns, ${ }^{*},{ }^{* *}$ : não significativo, significativo a $5 \%$ e a $1 \%$ de probabilidade respectivamente. As médias seguidas de letras iguais na vertical não diferem entre si, pelo teste de Tukey, a $5 \%$ de probabilidade. $\left({ }^{1}\right)$ Coeficiente de variação do tratamento principal. $\left({ }^{2}\right)$ Coeficiente de variação do subtratamento.

Tabela 2. Valor de F e coeficientes de variação dos fatores cultivar e do tempo de cultivo sobre os teores de micronutrientes das folhas das mudas de goiabeira

\begin{tabular}{|c|c|c|c|c|c|}
\hline Fatores & B & $\mathrm{Cu}$ & $\mathrm{Fe}$ & $\mathrm{Mn}$ & $\mathrm{Zn}$ \\
\hline & & & -Teste $\mathrm{F}$ & & \\
\hline Cultivar & $0,15^{\mathrm{ns}}$ & $52,07^{* *}$ & $3,26^{\mathrm{ns}}$ & $285,63^{* *}$ & $57,80^{* *}$ \\
\hline Tempo de cultivo & $114,03^{* *}$ & $40,60^{* *}$ & $56,83^{* *}$ & $6,43^{* *}$ & $4,27^{* *}$ \\
\hline Interação & $3,49^{*}$ & $15,73^{* *}$ & $10,01^{* *}$ & $8,06^{* *}$ & $4,04^{* *}$ \\
\hline Paluma & 37,7 & $6 \mathrm{~b}$ & 118 & 159 a & 33 a \\
\hline Século XXI & 37,1 & $7 \mathrm{a}$ & 122 & $107 \mathrm{~b}$ & $30 \mathrm{~b}$ \\
\hline C.V. $(\%)^{1}$ & 13,7 & 9,2 & 6,2 & 7,6 & 4,4 \\
\hline C.V. $(\%)^{2}$ & 8,2 & 13,6 & 7,7 & 7,5 & 10,8 \\
\hline
\end{tabular}

ns, *, **: não significativo, significativo a $5 \%$ e a $1 \%$ de probabilidade respectivamente. $\left({ }^{1}\right)$ C.V. da Parcela. $\left({ }^{2}\right)$ C.V. da subparcela.

Tabela 3. Estudos de regressão sobre os efeitos do tempo de cultivo no teor de micronutrientes das folhas das mudas de goiabeira (cv. Paluma e Século XXI)

\begin{tabular}{|c|c|c|c|c|c|c|}
\hline Nutriente & Paluma & Teste F & $\mathrm{R}^{2}$ & Século XXI & Teste F & $\mathrm{R}^{2}$ \\
\hline B & $Y=92,36-1,2349 x+0,0058 x^{2}$ & $95,85^{* *}$ & 0,99 & $Y=110,74-1,8688 x+0,0102 x^{2}$ & $127,51^{* *}$ & 0,94 \\
\hline $\mathrm{Cu}$ & $Y=20,762-0,3645 x+0,0019 x^{2}$ & $335,23^{* *}$ & 0,91 & $Y=7,1429+0,0505 x-0,0006 x^{2}$ & $116,03^{* *}$ & 0,81 \\
\hline $\mathrm{Fe}$ & $Y=9,85+2,269 x-0,0095 x^{2}$ & $85,40 * *$ & 0,82 & $Y=-15,381+3,6183 x-0,0205 x^{2}$ & $214,44^{* *}$ & 0,69 \\
\hline Mn & $Y=190,87-0,4198 x$ & $99,98^{* *}$ & 0,63 & $Y=170,60-2,004 x+0,0133 x^{2}$ & $129,37^{* *}$ & 0,68 \\
\hline $\mathrm{Zn}$ & $Y=17,48+0,5275 x-0,0036 x^{2}$ & $86,22 * *$ & 0,45 & $Y=43,52-0,3079 x+0,0015 x^{2}$ & $298,74^{* *}$ & 0,84 \\
\hline
\end{tabular}

${ }^{*},{ }^{* *}$ : Significativo a 5 e $1 \%$ respectivamente.

Com relação ao acúmulo de micronutrientes das mudas de goiabeira, verificou-se que houve diferença significativa entre as cultivares, tanto nas folhas para $\mathrm{B}, \mathrm{Cu}$, Fe e $\mathrm{Zn}$, no caule para $\mathrm{Cu}, \mathrm{Fe}, \mathrm{Mn}$ e $\mathrm{Zn}$ e também nas raízes para $\mathrm{B}, \mathrm{Cu}, \mathrm{Fe}, \mathrm{Mn}$ e $\mathrm{Zn}$
(Tabela 4). Na cv. Século XXI observou-se maior acúmulo de nutrientes que na cv. Paluma, no fim do período experimental nas folhas para $\mathrm{B}, \mathrm{Cu}, \mathrm{Fe}$ e $\mathrm{Zn}$, no caule para $\mathrm{Cu}$, Fe e $\mathrm{Zn}$ e nas raízes para $\mathrm{Mn}$ e $\mathrm{Zn}$ (Tabela 5). 
Com o tempo de cultivo, ocorreram diferenças no acúmulo de micronutrientes das folhas, do caule e das raízes (Tabela 4), sendo observado aumento do acúmulo de micronutrientes nos órgãos das mudas de goiabeira.

Observaram-se os seguintes valores para o acúmulo de micronutrientes das folhas, em $\mu \mathrm{g}$ por planta, $\mathrm{B}=450 ; \mathrm{Cu}=69 ; \mathrm{Fe}=1830 ; \mathrm{Mn}=1905 ; \mathrm{Zn}=424$, do caule, $\mathrm{B}=131 ; \mathrm{Cu}=41 ; \mathrm{Fe}=435 ; \mathrm{Mn}=525 ; \mathrm{Zn}=186$, e das raízes $B=127 ; \mathrm{Cu}=36 ; \mathrm{Fe}=4554 ; \mathrm{Mn}=950$ e $\mathrm{Zn}=269$. SALVADOR et al. (1999), estudando a omissão de macronutrientes, verificaram no tratamento completo com mudas obtidas de propagação via semente, após 135 dias de cultivo hidropônico, os seguintes valores nas folhas, em $\mu \mathrm{g}$ por planta, $\mathrm{B}=1303 ; \mathrm{Cu}=56$; $\mathrm{Fe}=5538 ; \mathrm{Mn}=6819 ; \mathrm{Zn}=1269$, do caule, $\mathrm{B}=473$; $\mathrm{Cu}=79 ; \mathrm{Fe}=1510 ; \mathrm{Mn}=7769 ; \mathrm{Zn}=877$, e das raízes $\mathrm{B}=513 ; \mathrm{Cu}=109 ; \mathrm{Fe}=1495 ; \mathrm{Mn}=6610$ e $\mathrm{Zn}=1153$. Possivelmente, essas variações no acúmulo de nutrientes pelas plantas de goiaba são justificadas pelo uso de solução nutritivas e material genético distintos, pois no trabalho de SALVADOR et al. (1999) utilizou-se solução de Hoagland e Arnon e plantas de goiaba propagadas de sementes.

Tabela 4. Valor de F e coeficientes de variação dos fatores cultivar e tempo de cultivo sobre acúmulo de micronutrientes nos diferentes órgãos das mudas de goiabeira

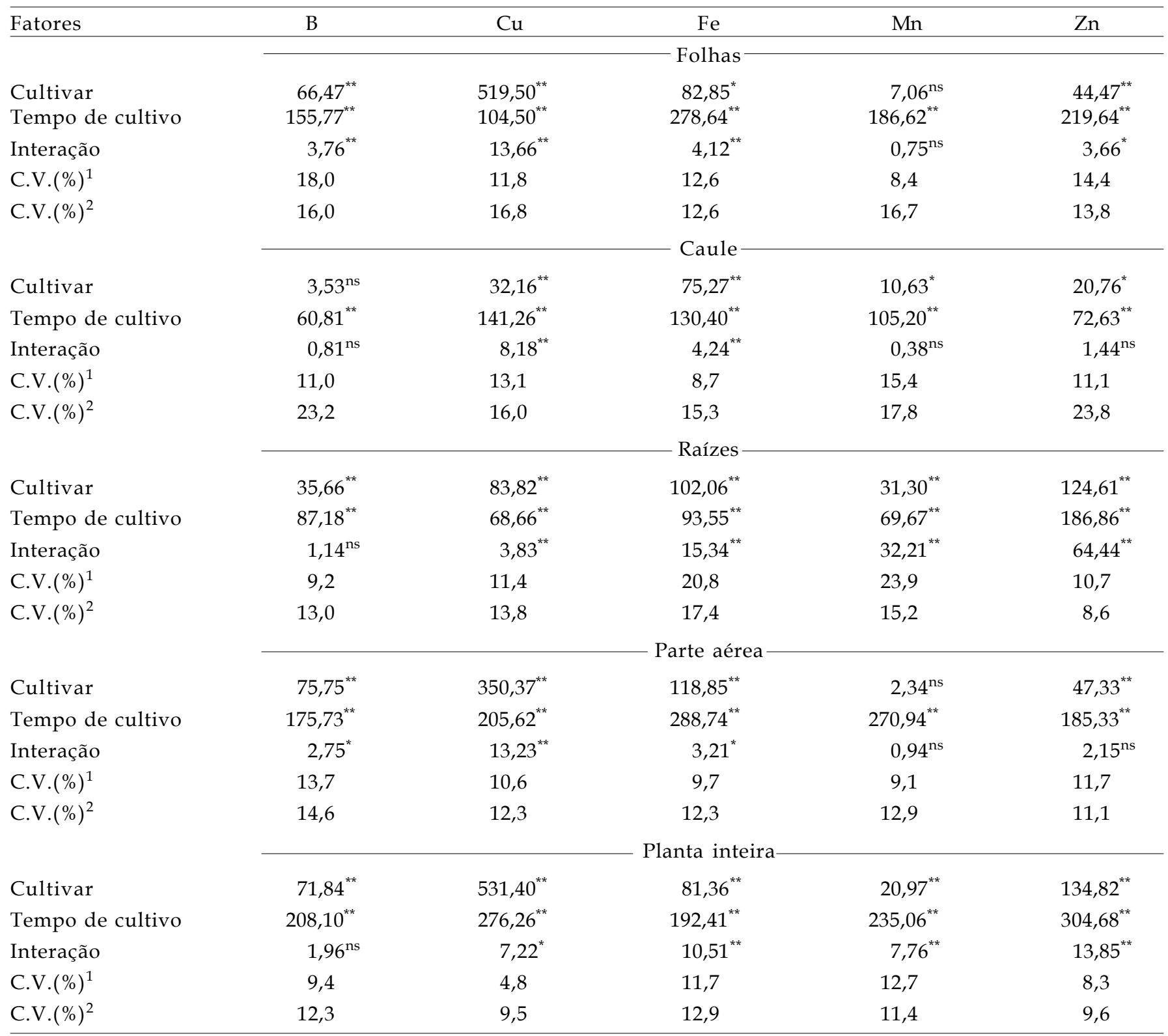

ns, * ** : não significativo, significativo a $5 \%$ e a $1 \%$ de probabilidade respectivamente. $\left({ }^{1 \text { e }}{ }^{2}\right.$ ) Coeficiente de variação da cultivar e do tempo de cultivo respectivamente. 
Tabela 5. Estudos de regressão sobre os efeitos do tempo de cultivo no acúmulo de micronutrientes das mudas de goiabeira (cv. Paluma e Século XXI)

\begin{tabular}{|c|c|c|c|c|c|c|}
\hline Nutriente & Paluma & Teste F & $R^{2}$ & Século XXI & Teste F & $\mathrm{R}^{2}$ \\
\hline & \multicolumn{6}{|c|}{ Folhas } \\
\hline B & $y=71,89-3,173 x+0,0467 x^{2}$ & $40,67^{* *}$ & 0,99 & $y=154,04-4,324 x+0,0598 x^{2}$ & $44,86^{* *}$ & 0,96 \\
\hline $\mathrm{Cu}$ & $y=31,64-1,143 x+0,011 x^{2}$ & $213,90^{* *}$ & 0,89 & $y=-13,36+0,75 x$ & $260,59^{* *}$ & 0,95 \\
\hline $\mathrm{Fe}$ & $y=192,01-13,912 x+0,2322 x^{2}$ & $53,71^{* *}$ & 0,96 & $y=-391,77+11,593 x+0,0723 x^{2}$ & $26,98^{*}$ & 0,97 \\
\hline$\square \mathrm{Mn}$ & $y=362,28-15,714 x+0,2353 x^{2}$ & $102,88^{* *}$ & 0,98 & & & \\
\hline \multirow[t]{2}{*}{$\mathrm{Zn}$} & $y=62,34-3,3275 x+0,0519 x^{2}$ & $48,30^{* *}$ & 0,99 & $y=-10,59+0,6276 x+0,0257 x^{2}$ & $16,48^{* *}$ & 0,98 \\
\hline & \multicolumn{6}{|c|}{ Caule } \\
\hline$\square \mathrm{B}$ & $y=78,52-2,223 x+0,0214 x^{2}$ & $120,38^{* *}$ & 0,94 & $\square$ & & \\
\hline $\mathrm{Cu}$ & $y=12,35-0,344 x+0,0044 x^{2}$ & $39,69^{* *}$ & 0,94 & $y=13,87-0,462 x+0,0062 x^{2}$ & $56,40^{* *}$ & 0,98 \\
\hline $\mathrm{Fe}$ & $y=-109,05+4,22 x$ & $342,89^{* *}$ & 0,97 & $y=-180,45+5,9023 x$ & $485,36^{* *}$ & 0,95 \\
\hline Mn & $y=74,98-1,612 x+0,0459 x^{2}$ & $10,02^{* *}$ & 0,99 & $y=76,26-2,66 x+0,0521 x^{2}$ & $50,61^{* *}$ & 0,98 \\
\hline \multirow[t]{2}{*}{$\mathrm{Zn}$} & $y=12,51-0,449 x+0,0147 x^{2}$ & $7,56^{*}$ & 0,99 & $y=8,32-0,633 x+0,0194 x^{2}$ & $16,09^{* *}$ & 0,99 \\
\hline & \multicolumn{6}{|c|}{ Raízes } \\
\hline B & $y=59,09-1,028 x+0,0134 x^{2}$ & $26,01^{* *}$ & 0,94 & $y=52,97-0,916 x+0,0114 x^{2}$ & $41,55^{* *}$ & 0,93 \\
\hline $\mathrm{Cu}$ & $y=-0,717+0,3147 x$ & $184,26^{* *}$ & 0,94 & $y=8,03-0,057 x+0,002 x^{2}$ & $12,34^{* *}$ & 0,90 \\
\hline $\mathrm{Fe}$ & $y=-1703,3+59,849 x$ & $267,21^{* *}$ & 0,94 & $y=730-15,319 x+0,2819 x^{2}$ & $34,03^{* *}$ & 0,97 \\
\hline $\mathrm{Mn}$ & $y=-160,37+15,634 x-0,0838 x^{2}$ & $17,52^{* *}$ & 0,84 & $y=-403,71+14,831 x$ & $297,49^{* *}$ & 0,94 \\
\hline \multirow[t]{2}{*}{$\mathrm{Zn}$} & $y=-5,84+3,0372 x-0,013 x^{2}$ & $9,42^{* *}$ & 0,93 & $y=-130,99+5,201 x-0,0092 x^{2}$ & $4,80^{*}$ & 0,96 \\
\hline & \multicolumn{6}{|c|}{ Parte aérea } \\
\hline B & $y=149,57-5,308 x+0,067 x^{2}$ & $56,67^{* *}$ & 0,98 & $y=233,39-6,636 x+0,0824 x^{2}$ & $70,25^{* *}$ & 0,96 \\
\hline $\mathrm{Cu}$ & $y=43,96-1,487 x+0,0154 x^{2}$ & $322,70^{* *}$ & 0,97 & $y=-29,22+1,2206 x$ & $490,66^{* *}$ & 0,97 \\
\hline $\mathrm{Fe}$ & $y=86,82-9,815 x+0,233 x^{2}$ & $36,37^{* *}$ & 0,96 & $y=-504,93+15,36 x+0,0866 x^{2}$ & $7,87^{*}$ & 0,97 \\
\hline$\square \mathrm{Mn}$ & $y=353,65-14,73 x+0,2635 x^{2}$ & $61,67^{* *}$ & 0,99 & $\square$ & & \\
\hline $\mathrm{Zn}$ & $y=74,86-3,777 x+0,0666 x^{2}$ & $30,18^{* *}$ & 0,99 & $y=-7,36+0,0468 x+0,0451 x^{2}$ & $26,28^{* *}$ & 0,99 \\
\hline
\end{tabular}

O acúmulo de Zn das raízes para a cv. Paluma foi de $176 \mu \mathrm{g}$ por planta, enquanto NATALE et al. (2002) observaram que mudas de goiabeira cv. Paluma cultivadas por 135 dias em vaso com solo obtiveram maior acúmulo de $\mathrm{Zn}$ nas raízes (233 $\mu \mathrm{g}$ por planta).

$\mathrm{O}$ acúmulo de micronutrientes pelas mudas corresponde à necessidade total de nutrientes pelas mudas, de forma que houve diferença para a cultivar na parte aérea e na planta inteira (Tabela 3), observandose na cv. Paluma maior acúmulo na parte aérea que na cv. Século XXI, para B, Cu, Fe e Zn, e na planta inteira para $\mathrm{B}, \mathrm{Cu}, \mathrm{Mn}$ e $\mathrm{Zn}$, no fim do período experimental (Tabela 5). Ao longo de período de cultivo das mudas de goiabeira, foram observados aumentos no acúmulo de micronutrientes nos diferentes órgãos das mudas (Tabela 5) e com reflexos na planta inteira (Figura 2).

O acúmulo de zinco na parte aérea das mudas da cultivar Paluma foi de $584 \mu \mathrm{g}$ de $\mathrm{Zn}$ por planta, enquanto NATALE et al. (2002) observaram menor acúmulo desse nutriente pelas mudas de goiabeira, cultivado em vasos preenchidos com solo (381 $\mu \mathrm{g}$ de $\mathrm{Zn}$ por planta). Essas diferenças possivelmente ocorreram devido ao sistema de cultivo, em solo e em solução nutritiva.

As mudas de goiabeira atingiram metade do acúmulo de micronutrientes entre 82 e 92 e 76 e 91 dias, respectivamente, para a cv. Paluma e Século XXI, acompanhando o acúmulo de massa seca nos diferentes órgãos, que atingiram a metade da massa seca total entre 75 e 94 e 80 e 90 dias para a cv. Paluma e Século XXI respectivamente. BARBOSA et al. (2003), estudando absorção de micronutrientes em mudas de gravioleira, concluíram que foi acumulado um terço do total desses nutrientes até metade do cultivo e o restante após este período. Teсchio et al. (2006), em mudas de citros, verificaram que para o Fe e Mn a maior exigência ocorre no período inicial, enquanto para $\mathrm{B}$ e $\mathrm{Zn}$, em um período intermediário e em $\mathrm{Cu}$, no final. As diferenças no acúmulo de nutrientes pelas 
plantas devem-se aos parâmetros cinéticos de absorção dos nutrientes, pois tem influência genética e estão relacionadas às características morfológicas e fisiológicas da planta (MARSCHNER, 1995).

Acrescenta-se, ainda, que nas mudas de goiabeira da cv. Paluma houve acúmulo dos micronutrientes na seguinte quantidade: 632; 134; 8103; 3052 e $760 \mu \mathrm{g}$ por planta para B, Cu, Fe, Mn e Zn, enquanto a cv. Século XXI acumulou 783; 158; 5534; 3709 e 997 ìg por planta para B, $\mathrm{Cu}, \mathrm{Fe}, \mathrm{Mn}$ e $\mathrm{Zn}$ respectivamente (Figura 3). SALVADOR et al. (1999) observaram, no tratamento completo, 708; 146; 6818; 3380 e $879 \mu \mathrm{g}$ por planta para B, Cu, Fe, Mn e Zn respectivamente.

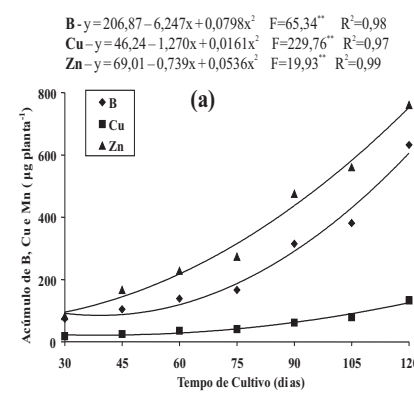

$$
\begin{array}{lll}
\mathrm{Fe}-\mathrm{y}=-811,86+24,491 \mathrm{x}+0,4033 \mathrm{x}^{2} & F=7,56 & R^{2}=0,98 \\
\mathrm{Mn}-\mathrm{y}=233,52-1,544 \mathrm{x}+0,199 x^{2} & F=18,82^{2} & R^{2}=0,97
\end{array}
$$

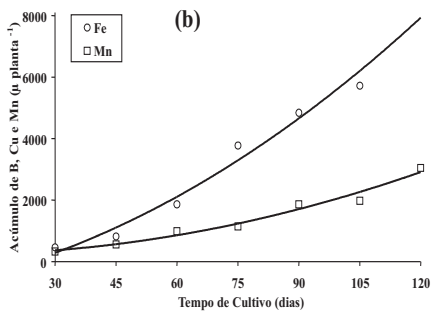

B $-y=284,27-7,532 x+0,0938 x^{2} \quad F=89,83^{\prime \prime} \quad R^{2}=0,96$ Cu $-y=-6,49+0,697 x+0,0051 x^{2} \quad F=5,53 \quad R^{2}=0,98$
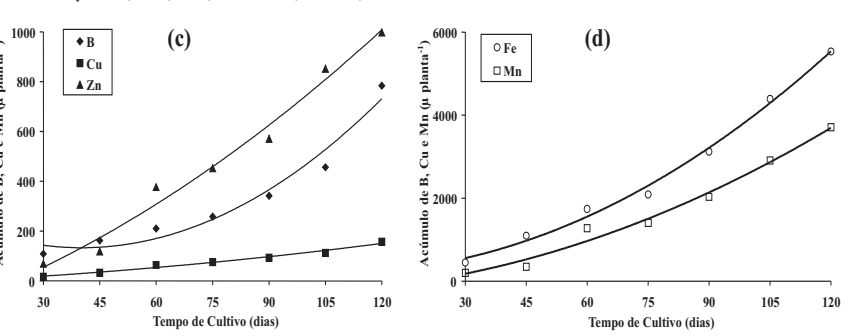
Fe $-y=225,58+0,024 x+0,3686 x^{2} \quad F=37,21 * \quad R^{2}=0,99$

Figura 2. Efeito do tempo de cultivo no acúmulo de micronutrientes nas mudas de goiabeira cultivares Paluma (a e b) e Século XXI (c e d) cultivadas em solução nutritiva durante 120 dias após o transplantio (médias de quatro plantas).

De maneira geral, a quantidade de micronutrientes está próxima da encontrada por SALVADOR et al. (1999), mesmo considerando a diferença de material genético e condições distintas de cultivo. Portanto, por esses resultados verifica-se que a exigência nutricional em micronutrientes de mudas de goiabeira pode variar em função da cultivar utilizada. De forma geral, o acúmulo médio de micronutrientes pelas mudas de goiabeira (média de duas cultivares) obedeceu à seguinte seqüência: $\mathrm{Fe}>\mathrm{Mn}>\mathrm{Zn}>\mathrm{B}>\mathrm{Cu}$ (Figura 2).

Observa-se que o acúmulo de micronutrientes nas mudas da cv. Paluma aumentou rapidamente a partir dos 45 dias para o Fe e dos 75 dias para B, Cu, $\mathrm{Mn}$ e $\mathrm{Zn}$, enquanto na cv. Século XXI, esse aumento ocorreu a partir dos 45 dias para $\mathrm{Cu}, \mathrm{Mn}$ e $\mathrm{Zn}$, dos 75 dias para $\mathrm{Fe}$ e dos 105 dias para o B (Figura 2).
Considerando a distribuição (em porcentagem do total acumulado) dos micronutrientes por mudas de goiabeira (média de duas cultivares) ao longo do tempo (Tabela 6), observa-se que o acúmulo do B é mais lento que os demais. A presente distribuição dos nutrientes acumulados ao longo do tempo poderá auxiliar programas de adubação das mudas, entretanto, deve-se salientar que as quantidades sugeridas de micronutrientes devem ser acrescidas de um fator de eficiência de aproveitamento.

Tabela 6. Distribuição (em porcentagem do total) do acúmulo de micronutrientes ao longo do cultivo de mudas de goiabeira

\begin{tabular}{lrrrrr}
\hline Tempo de cultivo & $\mathrm{B}$ & $\mathrm{Cu}$ & $\mathrm{Fe}$ & $\mathrm{Mn}$ & $\mathrm{Zn}$ \\
\cline { 2 - 6 } 30 & 12,9 & 11,8 & 6,7 & 7,8 & 8,6 \\
45 & 18,8 & 19,7 & 14,1 & 13,5 & 16,2 \\
60 & 24,6 & 34,1 & 26,4 & 33,4 & 34,4 \\
75 & 30,0 & 40,0 & 43,0 & 37,7 & 41,3 \\
90 & 46,4 & 53,1 & 58,4 & 57,6 & 59,5 \\
105 & 59,2 & 65,7 & 74,1 & 72,3 & 80,3 \\
120 & 100,0 & 100,0 & 100,0 & 100,0 & 100,0 \\
\hline
\end{tabular}

Do total de nutrientes absorvidos, observa-se na cv. Século XXI maior incremento em relação à cv. Paluma, para B (24\%), Cu (18\%), Mn (22\%) e Zn (33\%); entretanto, na cv. Paluma a absorção de Fe foi $46 \%$ maior que na Século XXI (Figura 2). Portanto, pode-se destacar que houve acúmulo de micronutrientes nas mudas de goiabeira estudadas, e a maior variação foi para ferro e zinco.

Pode-se observar que o acúmulo de $\mathrm{B}, \mathrm{Cu}, \mathrm{Mn}$ e $\mathrm{Zn}$ foi maior nas folhas, enquanto o Fe foi maior nas raízes das mudas de goiabeira (Figura 3). Resultados semelhantes foram observados por SALVADOR et al. (1999) com o tratamento completo. Essas diferenças, especialmente nas raízes, se devem ao material genético e ao tempo de cultivo distintos. Em plantas adultas, a exemplo do citros, os micronutrientes estão mais concentrados nos frutos e nas raízes em detrimento das folhas e do caule (MATTOS JÚNior et al., 2003).
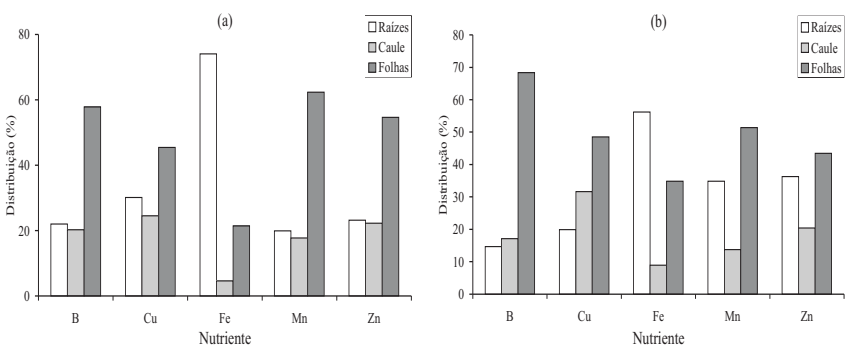

Figura 3. Acúmulo de micronutrientes (\%) nos órgãos das mudas de goiabeira cultivares Paluma (a) e Século XXI (b) cultivadas em solução nutritiva durante 120 dias após o transplantio (médias de quatro plantas). 


\section{CONCLUSÕES}

1. A muda de goiabeira da cultivar Século XXI foi mais exigente em micronutrientes que as mudas da cultivar Paluma.

2. O acúmulo médio de micronutrientes pelas mudas de goiabeira obedeceu à seguinte seqüência: $\mathrm{Fe}>\mathrm{Mn}>\mathrm{Zn}>\mathrm{B}>\mathrm{Cu}$, sendo maior nas folhas para $\mathrm{B}, \mathrm{Cu}$, Mn e $Z n$, e nas raízes para Fe.

3. O acúmulo de micronutrientes para as cultivares Paluma e Século XXI em ìg por planta foi de: $\mathrm{B}$ (632 e 783), Cu (134 e 158), Fe (8103 e 5534), Mn (3052 e 3709) e Zn (760 e 997) respectivamente.

\section{AGRADECIMENTOS}

À FAPESP pelo apoio financeiro concedido (Processo 2003/11649-6 - Auxílio à Pesquisa).

\section{REFERÊNCIAS}

AGRIANUAL: Anuário da agricultura brasileira. São Paulo: FNP Consultoria e Comércio, 2006. p. 332.

ANUÁRIO IEA. Série de Informações Estatísticas da Agricultura. São Paulo, 2006, v.17, n.1, p.1-116.

BARBOSA, Z., SOARES, I., CRISÓTOMO, L.A. Crescimento e absorção de nutrientes por mudas de gravioleira. Revista Brasileira de Fruticultura, Jaboticabal, v.25, n.3, p.519-522, 2003.

BATAGLIA, A.C.; FURLANI, A.M.C.; TEIXEIRA,J.P.F.; FURLANI, P.R.;GALLO, J.R. Métodos de análise química de plantas. Campinas: Instituto Agronômico, 1983. 48p. (Boletim Técnico, 78).

CASTELLANE, P.D.; ARAÚJO, J.A.C. de. Cultivo sem solo: hidroponia. 4.ed. Jaboticabal: FUNEP, 1995. 43p.

FRANCO, C.F., PRADO, R.M. Uso de soluções nutritivas no desenvolvimento e no estado nutricional de mudas de goiabeira: macronutrientes Acta Scientiarum Agronomia, Maringá, v.28, n.2, p.199-205, 2006.

MALAVOLTA, E.; VITTI, G.C.; OLIVEIRA, S.A. Avaliação do estado nutricional das plantas: princípios e aplicações. 2.ed. Piracicaba: POTAFÓS, 1997.319p.

MARSCHNER, H. Mineral nutrition of higher plants. 2th. London: Academic Press, 1995. 889p.

MATTOS JÚNIOR, D.; QUAGGIO, J.A.; CANTARELLA, H.; ALVA, A.K. Nutrient content of biomass components of Hamlin sweet orange trees. Scientia Agricola, Piracicaba, v.60, n.1, p.155-160, 2003.

NATALE, W.; PRADO, R.M.; CORRÊA, M.C.M.; SILVA, M.A.C.; PEREIRA, L. Resposta de mudas de goiabeira à aplicação de zinco. Revista Brasileira de Fruticultura, Jaboticabal, v.24, n.3, p.770-773, 2002.
NATALE, W.; COUTINHO, E.L.M.; BOARETTO, A.E.; PEREIRA, F.M. Goiabeira: calagem e adubação. Jaboticabal: Funep, 1996. 22p.

ROZANE, D.E.; COUTO, F.A.D.A. (Ed.). Cultura da Goiabeira: Tecnologia e Mercado. UFV, Viçosa, p.53-78, 2003.

SALVADOR, J.A., MOREIRA, A.; MURAOKA, T. Efeito da omissão combinada de N, P, K S nos teores foliares de macronutrientes em mudas de goiabeira. Scientia Agricola, Piracicaba, v.56, n.2, p.501-507,1999.

TÁVORA, F.J.A.F., FERREIRA, R.G.; HERNANDEZ, F.F.F. Crescimento e relações hídricas em plantas de goiabeira submetidas a estresse salino com $\mathrm{NaCl}$. Revista Brasileira de Fruticultura, Jaboticabal, v.23, n.2, p.441-446, 2001.

TECCHIO, M.A.; LEONEL, S; LIMA. C.P.; VILLAS BOAS, R.L.; ALMEIDA, E.L.P.; CORREAA, J.C. Crescimento e acúmulo de nutrientes no porta-enxerto citrumelo 'Swingle', cultivado em substrato. Bioscience Journal, Uberlândia, v.22, n.1, p.37$44,2006$. 